\title{
The need to develop alternative measures to seclusion and restraint
} Anu Putkonen

\author{
Address: Kuopio University, Faculty of Forensic Psychiatry, Niuvanniemi Hospital, 70240 Kuopio, Finland \\ from WPA Thematic Conference. Coercive Treatment in Psychiatry: A Comprehensive Review \\ Dresden, Germany. 6-8 June 2007 \\ Published: 19 December 2007 \\ BMC Psychiatry 2007, 7(Suppl I):S63 doi:10.1 186/I47I-244X-7-SI-S63
}

This abstract is available from: http://www.biomedcentral.com/I47I-244X/7/SI/S63

(C) 2007 Putkonen; licensee BioMed Central Ltd.

In studies of psychiatric inpatients, the incidence of seclusion or restraint $(\mathrm{S} / \mathrm{R})$ varies widely $(0-66 \%)$. Frequent use has been associated with e.g. violent behavior, age, sex, psychiatric symptoms and diagnosis of the patient, but also with the ward culture. The use of S/R is problematic since no randomized controlled studies of the safety or effectiveness of them were published. S/R may save lives and prevent injuries in acute violent or suicidal incidences. However, the indications and durations of the coercive methods vary widely between units. Considerable economical costs, and psychological and physical injuries of both patients and nurses associated with $S / R$ have been reported. In USA, S/R has been highly prioritized since 1998 when the publicity of over $140 \mathrm{~S} / \mathrm{R}$ related deaths lead to congressional hearings. Patients with psychiatric symptoms are particularly vulnerable for stress, and many of them have suffered from maltreatment. Frequent use of S/R may increase stress on the ward, harm therapeutic relations, the effect of medicines, and the recovery. Several countries, settings and nursing organizations (e.g. APNA) have projects committed to the reduction of S/R. Some promising prevention programs of violent behavior of psychiatric patients have been developed. Randomized controlled trials of $S / R$, and alternative methods for containment of violent behavior are needed. 